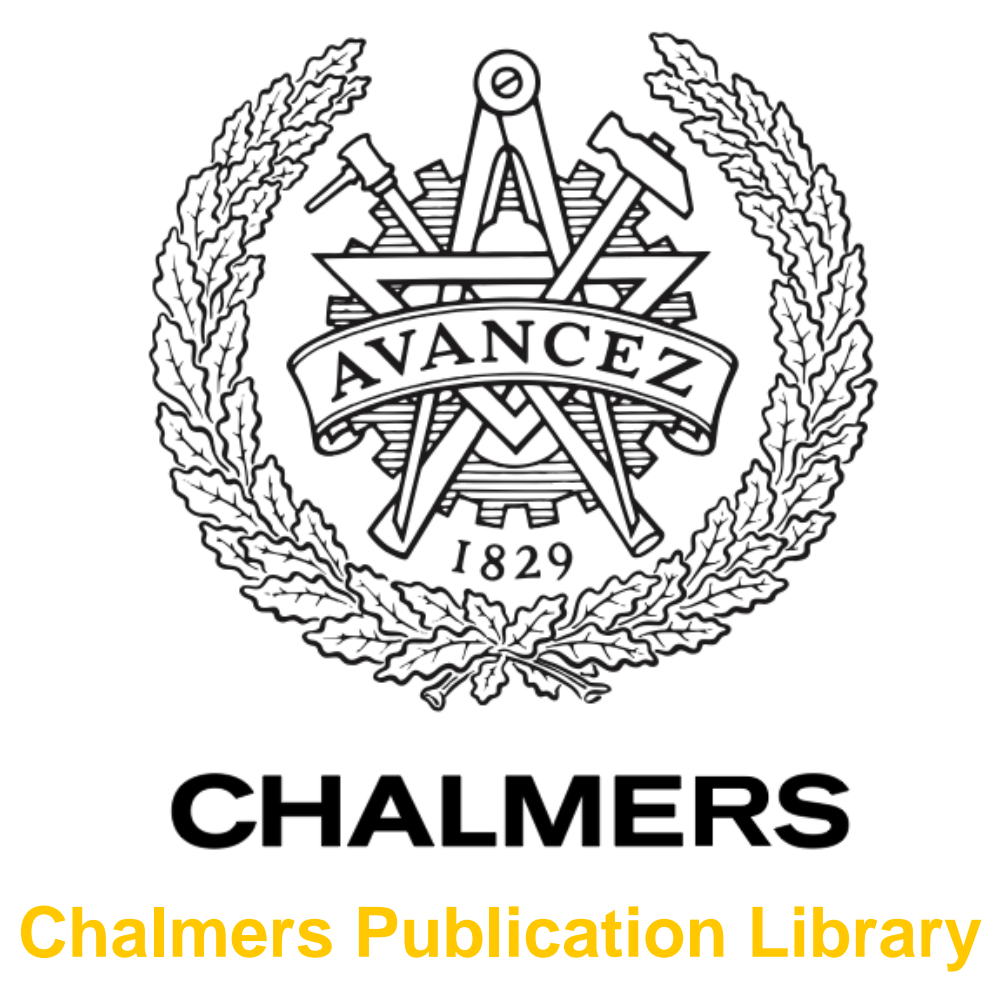

Multipath-assisted maximum-likelihood indoor positioning using UWB signals

This document has been downloaded from Chalmers Publication Library (CPL). It is the author's version of a work that was accepted for publication in:

IEEE ICC 2014 Workshop on Advances in Network Localization and Navigation (ANLN)

Citation for the published paper:

Leitinger, E. ; Fröhle, M. ; Meissner, P. (2014) "Multipath-assisted maximum-likelihood indoor positioning using UWB signals". IEEE ICC 2014 Workshop on Advances in Network Localization and Navigation (ANLN) pp. 170-175.

http://dx.doi.org/10.1109/ICCW.2014.6881191

Downloaded from: http://publications.lib.chalmers.se/publication/203646

Notice: Changes introduced as a result of publishing processes such as copy-editing and formatting may not be reflected in this document. For a definitive version of this work, please refer to the published source. Please note that access to the published version might require a subscription.

Chalmers Publication Library (CPL) offers the possibility of retrieving research publications produced at Chalmers University of Technology. It covers all types of publications: articles, dissertations, licentiate theses, masters theses, conference papers, reports etc. Since 2006 it is the official tool for Chalmers official publication statistics. To ensure that Chalmers research results are disseminated as widely as possible, an Open Access Policy has been adopted.

The CPL service is administrated and maintained by Chalmers Library. 


\title{
Multipath-Assisted Maximum-Likelihood Indoor Positioning using UWB Signals
}

\author{
Erik Leitinger*†, Markus Fröhle ${ }^{+\dagger}$, Paul Meissner*, and Klaus Witrisal* \\ * Email: erik.leitinger@tugraz.at, Graz University of Technology, Austria \\ + Email: frohle@chalmers.se, Chalmers University of Technology, Sweden , ${ }^{\dagger}$ equal contribution
}

\begin{abstract}
Multipath-assisted indoor positioning (using ultrawideband signals) exploits the geometric information contained in deterministic multipath components. With the help of a-priori available floorplan information, robust localization can be achieved, even in absence of a line-of-sight connection between anchor and agent. In a recent work, the Cramér-Rao lower bound has been derived for the position estimation variance using a channel model which explicitly takes into account diffuse multipath as a stochastic noise process in addition to the deterministic multipath components. In this paper, we adapt this model for position estimation via a measurement likelihood function and evaluate the performance for real channel measurements. Performance results confirm the applicability of this approach. A position accuracy better than $2.5 \mathrm{~cm}$ has been obtained in $90 \%$ of the estimates using only one active anchor at a bandwidth of $2 \mathrm{GHz}$ and robustness against non-line-of-sight situations has been demonstrated.
\end{abstract}

\section{INTRODUCTION}

Ultra-wideband (UWB) signals are promising candidates for indoor positioning. Their large frequency range offers a fine delay resolution and robustness in harsh propagation environments such as indoors [1]. The benefit of the UWB channel is that many of the multipath components (MPCs) are recognizable and resolvable in the measurements.

Deterministic MPCs, having parameters that can be modeled as a function of the surrounding geometry, can be used for localization and tracking [2]-[5]. The transmitted signal which is reflected at e.g. a wall, can be seen as being emitted from a virtual source located behind the reflecting surface. With the help of a floorplan, it is possible to calculate the position of these virtual sources and use them as so-called virtual anchors (VAs) for localization. Fig. 1 illustrates an exemplary floor-plan with two fixed anchors and a small subset of corresponding VAs. In this way, localization is possible with only a single anchor node independent of a line-of-sight (LOS) or non-LOS (NLOS) situation. We call this approach multipath-assisted indoor navigation and tracking (MINT). In [5], the Cramér-Rao Lower Bound (CRLB) has been derived for this problem. Diffuse multipath (DM), e.g. scattered signals which are not covered by the deterministic model, impairs the detection of the useful deterministic components. This

This work was supported by the Austrian Science Fund (FWF) within the National Research Network SISE project S10610, by the Austrian Research Promotion Agency (FFG) within KIRAS PL3, grant no. 832335 "LOBSTER", and by EU FP7 Marie Curie Initial Training Network MULTI-POS (Multitechnology Positioning Professionals) under grant nr. 316528.

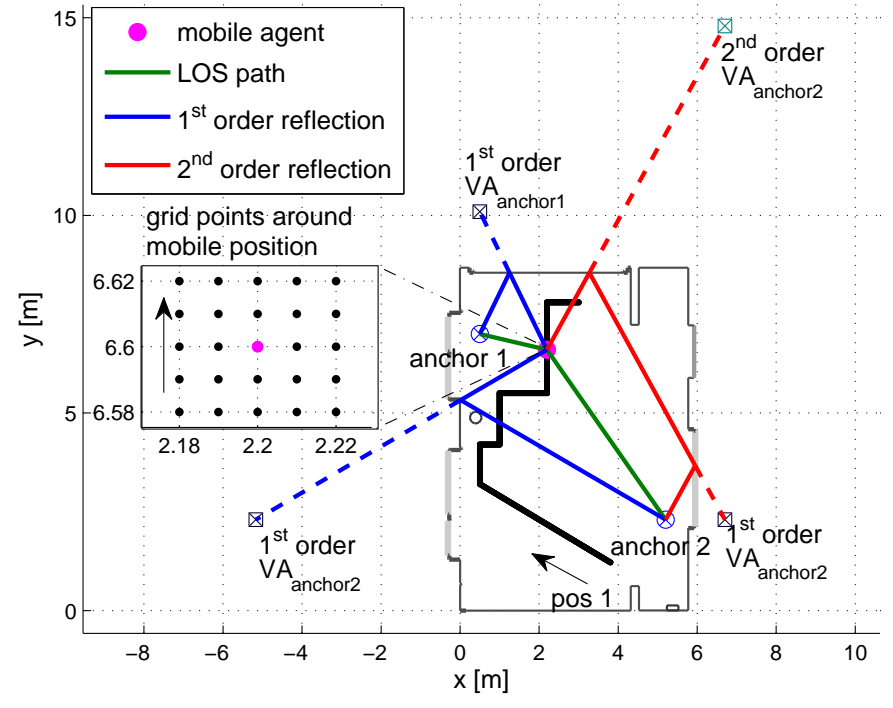

Fig. 1. Floor-plan of an empty seminar room with a 5-cm-spaced trajectory (black line) of agent positions. The close-up shows that for every agent position $\mathbf{p}$ (magenta dot), a set of 25 measurements has been recorded in a rectangular grid with $1 \mathrm{~cm}$ spacing. Furthermore, two physical anchors $j=\{1,2\}$ are illustrated and a few of the expected virtual anchors (VAs).

interfering part of the channel is modeled as an additive stochastic process.

In this paper, we adapt the model of [5] to formulate the maximum likelihood estimator (MLE) of the agent position. We also evaluate the localization performance with real measurements, where we analyze in particular the impact of considering DM in the likelihood function (LHF). The LHF is highly multi-modal and the MLE problem is non-convex, meaning that methods such as gradient descent are not useful for finding the global maximum. We thus apply particle filter methods [6] with swarm behavior [7]. The contributions of this work are:

- Formulation of MLE for the channel model given in [5]

- Localization performance evaluation of the MLE with real indoor channel measurements

- Characterization of the DM, i.e. estimation of its power delay profile (PDP), and analysis of its impact on the MLE, and

- Comparison between the MLE co-variance and the estimated CRLB. 


\section{Problem OVERVIEW}

\section{A. Signal Model}

A UWB baseband signal $s(t) \in \mathbb{R}$ with effective pulse duration $T_{p}$, is transmitted from an anchor $j=1 \ldots N_{\mathrm{A}}$, located at position $\mathbf{p}_{1}^{(j)} \in \mathbb{R}^{2}$, where $N_{\mathrm{A}}$ is the number of physical anchors considered. The received signal at the agent position $\mathbf{p}$ is modeled as [5]

$$
\begin{aligned}
r^{(j)}(t) & =r_{\mathrm{det}}^{(j)}(t)+r_{\mathrm{diff}}^{(j)}(t)+w(t) \\
& =\sum_{k=1}^{K^{(j)}} \alpha_{k}^{(j)} s\left(t-\tau_{k}^{(j)}\right)+s(t) * \nu^{(j)}(t)+w(t),
\end{aligned}
$$

where the first term comprises a sum of $K^{(j)}$ deterministic MPCs with complex amplitudes $\alpha_{k}^{(j)} \in \mathbb{C}$ and delays $\tau_{k}^{(j)}=\frac{1}{c}\left\|\mathbf{p}-\mathbf{p}_{k}^{(j)}\right\|_{2}$, where $c$ is the speed of light. Due to the knowledge of the floorplan, we can associate these MPCs to specular reflections at surfaces, i.e. they are modeled by VAs at positions $\mathbf{p}_{k}^{(j)}$, with $k=2 \ldots K^{(j)}$, where $K^{(j)}$ is the number of expected VAs at position p (c.f. Fig. 1). The second term $r_{\text {diff }}^{(j)}(t)$ denotes the convolution $(*)$ of the transmitted signal $s(t)$ with the $\mathrm{DM} \nu^{(j)}(t)$ which is modeled as a zero-mean Gaussian random process. We assume uncorrelated scattering along the delay axis $\tau$. Hence, the auto-correlation function (ACF) of $\nu^{(j)}(t)$ is given by $C_{\nu}^{(j)}(\tau, u)=S_{\nu}^{(j)}(\tau) \delta(\tau-u)$, where $S_{\nu}^{(j)}(\tau)$ is the PDP of the DM for the $j$-th anchor at the agent position p. According to this model, the DM is quasistationary in the spatial domain, which means that $S_{\nu}^{(j)}(\tau)$ does not change in the vicinity of position $\mathbf{p}$ [1]. Note that $r_{\text {diff }}^{(j)}(t)$ is non-stationary in the delay domain. Finally, the last term $w(t)$ denotes AWGN with a double-sided power spectral density (PSD) of $N_{0}$.

In Fig. 2, a set of measured signals $\left\{r_{i}^{(j)}(t)\right\}_{i=1}^{25}$ is shown for anchor 2 (colored thin lines). The measurements $r_{i}^{(j)}(t)$ are from the direct vicinity of the agent's actual position $\mathbf{p}$ to guarantee a quasi-stationary behavior in the spatial domain, as illustrated in Fig. 1. Further, the correlation function of the DM plus AWGN is illustrated and given by $C^{(j)}(t, u)=$ $\int_{-\infty}^{\infty} S_{\nu}^{(j)}(\tau) s(t-\tau) s(u-\tau) \mathrm{d} \tau+N_{0} \delta(t-u)$ (bold dashed red line). The received signal $r^{(j)}(t)$ at the agent's actual position $\mathbf{p}$ (i.e. at the center of the grid) is illustrated by the bold dashed black line.

\section{B. Likelihood Function (LHF)}

The LHF is defined for the channel parameter vector $\psi=$ $\left[\boldsymbol{\tau}^{\mathrm{T}},\left(\boldsymbol{\alpha}^{\mathrm{R}}\right)^{\mathrm{T}},\left(\boldsymbol{\alpha}^{\mathrm{I}}\right)^{\mathrm{T}}\right]^{\mathrm{T}}$, where $\boldsymbol{\tau}=\left[\tau_{1}, \ldots, \tau_{K}\right]^{\mathrm{T}}$ represents the vector with the geometry-related delays and $\left(\boldsymbol{\alpha}^{\mathrm{R}}\right)=$ $\left[\alpha_{1}^{R}, \ldots, \alpha_{K}^{R}\right]^{\mathrm{T}}, \quad\left(\boldsymbol{\alpha}^{\mathrm{I}}\right)=\left[\alpha_{1}^{I}, \ldots, \alpha_{K}^{I}\right]^{\mathrm{T}}$ are the real and imaginary parts of the corresponding complex amplitudes. An approximation of this LHF, using a whitening operation as

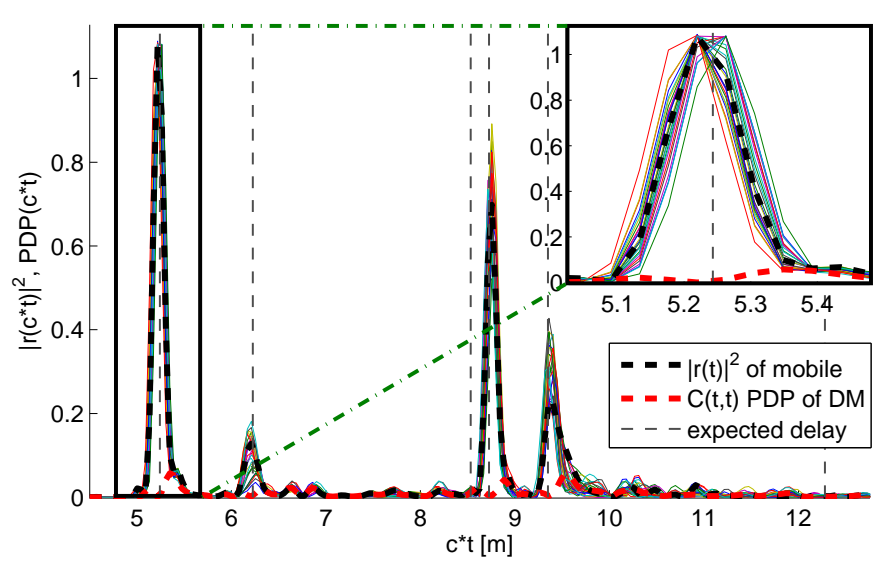

Fig. 2. A set of received $\left\{r_{i}^{(j)}(t)\right\}_{i=1}^{25}$ measurements recorded at grid positions illustrated in Fig. 1 (thin solid colored lines), including the actual agent position $\mathbf{p}=[2.2,6.6]^{\mathrm{T}}$ (bold dashed black line). Anchor 2 is used. Also, the corresponding DM correlation function $C^{(j)}(t, t)$ is shown (bold dashed red line). Expected delays to VAs visible at the agent's position are illustrated by dashed gray vertical lines.

described in [5], is given by

$$
\begin{aligned}
p\left(r^{(j)}(t) \mid \boldsymbol{\psi}\right) \propto \exp & \left(\frac{2}{N_{0}} \int_{0}^{T_{0}} \Re\left\{r^{(j)}(t) \sum_{k=1}^{K} w_{k}^{2} \alpha_{k}^{*} s\left(t-\tau_{k}\right)\right\} \mathrm{d} t\right. \\
& \left.-\frac{1}{N_{0}} \int_{0}^{T_{0}}\left|\sum_{k=1}^{K} w_{k} \alpha_{k} s\left(t-\tau_{k}\right)\right|^{2} \mathrm{~d} t\right)
\end{aligned}
$$

where $T_{0}$ is the observation time and $w_{k}^{2}=N_{0} /\left(N_{0}+\right.$ $\left.T_{p} S_{\nu}\left(\tau_{k}\right)\right)$ are weighting factors accounting for the DM [5]. The term $T_{p} S_{\nu}\left(\tau_{k}\right)$ represents the equivalent power of the DM. For the sake of brevity, we have dropped the anchor index $j$ for the channel parameters, but we will use it in situations where we emphasize the explicit anchor dependence.

The LHF for the sampled received signal, where $T_{s}$ represents the sampling time, is described by

$$
\begin{aligned}
p\left(\mathbf{r}^{(j)} \mid \boldsymbol{\psi}\right)=p\left(\mathbf{r}^{(j)} \mid \boldsymbol{\tau}, \boldsymbol{\alpha}\right) \propto \\
\quad \exp \left(-\frac{1}{2}\left(\mathbf{r}^{(j)}-\mathbf{S}^{\mathrm{H}} \boldsymbol{\alpha}\right)^{\mathrm{H}} \mathbf{C}^{-1}\left(\mathbf{r}^{(j)}-\mathbf{S}^{\mathrm{H}} \boldsymbol{\alpha}\right)\right)
\end{aligned}
$$

where $\mathbf{r}^{(j)}=\left[r^{(j)}\left(T_{s}\right), \ldots, r^{(j)}\left(M T_{s}\right)\right]^{\mathrm{T}} \in \mathbb{R}^{M}$ denotes the sampled received signal vector and $(\cdot)^{\mathrm{H}}$ is the Hermitian conjugate. The signal matrix $\mathbf{S} \in \mathbb{R}^{K \times M}$ is given by

$$
\mathbf{S}=\left[\begin{array}{cccc}
s\left(T_{s}-\tau_{1}\right) & s\left(2 T_{s}-\tau_{1}\right) & \ldots & s\left(M T_{s}-\tau_{1}\right) \\
\vdots & & & \vdots \\
s\left(T_{s}-\tau_{K}\right) & s\left(2 T_{s}-\tau_{K}\right) & \ldots & s\left(M T_{s}-\tau_{K}\right)
\end{array}\right]
$$

containing delayed versions of the transmitted pulse $s\left(n T_{s}-\right.$ $\left.\tau_{k}\right)$. In (3), the matrix $\mathbf{C}=N_{0} \mathbf{I}_{M}+\mathbf{K}_{\nu} \in \mathbb{R}^{M \times M}$ denotes the co-variance matrix of white noise and DM. The DM co-variance matrix is given by $\left[\mathbf{K}_{\nu}\right]_{n, m}=$ $T_{s} \sum_{i=1}^{M} S_{\nu}\left(i T_{s}\right) s\left(n T_{s}-i T_{s}\right) s\left(m T_{s}-i T_{s}\right)$, where $[\cdot]_{n, m}$ is the $(n, m)$-th matrix element. $\mathbf{I}_{M}$ is the identity matrix of size $M$. 


\section{Cramér-Rao Lower Bound (CRLB)}

As a performance measure and lower bound we use the CRLB of the position error defined by the inequality $\mathbb{E}\{\| \mathbf{p}-$ $\left.\hat{\mathbf{p}} \|\left.\right|_{2} ^{2}\right\} \geq \operatorname{tr}\left\{\mathbf{J}_{\mathbf{p}}^{-1}\right\}$, where $\mathbf{J}_{\mathbf{p}}$ is the equivalent Fisher information matrix (EFIM) [4] for the position vector and $\operatorname{tr}\{\cdot\}$ is the trace operator. In [5], the CRLB has been derived for the signal model in (2). The CRLB shows the influence of the VA positions, i.e. the room geometry, and of the power ratio between the modeled "information carrying" deterministic MPCs and detrimental DM, on the precision of the position estimator. With the assumption that the signals received from different propagation paths are orthogonal to one another, the EFIM $\mathbf{J}_{\mathbf{p}}$ is formulated for a set of anchors in a canonical form by

$$
\mathbf{J}_{\mathbf{p}}=\frac{8 \pi^{2} \beta^{2}}{c^{2}} \sum_{j=1}^{N_{\mathrm{A}}} \sum_{k=1}^{K^{(j)}} \operatorname{SINR}_{k}^{(j)} \mathbf{J}_{\mathrm{r}}\left(\phi_{k}^{(j)}\right),
$$

where $\beta$ denotes the effective signal bandwidth and $\mathbf{J}_{\mathrm{r}}\left(\phi_{k}^{(j)}\right)$ the ranging direction matrix, which has an eigenvector in direction $\phi_{k}^{(j)}$ from the agent to the $k$-th VA. $\operatorname{SINR}_{k}^{(j)}$ represents the signal-to-interference-plus-noise ratio of the $k$-th MPC and is defined as

$$
\operatorname{SINR}_{k}^{(j)}=\left(w_{k}^{(j)}\right)^{2} \frac{\left|\alpha_{k}^{(j)}\right|^{2}}{N_{0}}=\frac{\left|\alpha_{k}^{(j)}\right|^{2}}{N_{0}+T_{p} S_{\nu}^{(j)}\left(\tau_{k}\right)} .
$$

The value of the SINR shows the importance of the knowledge of the DM statistics in order to accurately estimate the information gained by a MPC.

\section{MLE of the agent's position}

The computation of the LHF can be seen as a position dependent channel estimation problem with prior floorplan information. Using the floorplan knowledge, the sets of VAs are computed for every anchor using optical ray-tracing [2]. In realistic scenarios, the floorplan has uncertainties which we take into account by using a probabilistic formulation of the VA positions. The maximum a-posteriori (MAP) estimator of the agent's position can then be formulated as

$$
\begin{aligned}
& \hat{\mathbf{p}}_{\mathrm{MAP}}=\underset{\mathbf{p}, \boldsymbol{\tau}, \boldsymbol{\alpha}}{\arg \max } \prod_{j=1}^{N_{\mathrm{A}}} \mathrm{p}\left(\mathbf{p}, \boldsymbol{\tau}, \boldsymbol{\alpha} \mid \mathbf{r}^{(j)}\right) \\
& =\underset{\mathbf{p}, \boldsymbol{\tau}, \boldsymbol{\alpha}}{\arg \max } \prod_{j=1}^{N_{\mathrm{A}}} \mathrm{p}\left(\mathbf{r}^{(j)} \mid \boldsymbol{\tau}, \boldsymbol{\alpha}\right) \mathrm{p}(\boldsymbol{\alpha} \mid \boldsymbol{\tau}) \mathrm{p}\left(\boldsymbol{\tau} \mid \mathbf{p}, \mathbf{p}_{\mathrm{VA}}^{(j)}\right) \mathrm{p}(\mathbf{p})
\end{aligned}
$$

where $\mathbf{p}_{\mathrm{VA}}^{(j)}=\left[\mathbf{p}_{1}^{(j)}, \ldots, \mathbf{p}_{K^{(j)}}^{(j)}\right]^{\mathbf{T}}$ is the set of VAs to compute the delay paths $\tau$ of the MPCs for the agent's position $\mathbf{p}$. If we assume that the VA positions are exactly known then the PDFs are $\mathrm{p}\left(\boldsymbol{\tau} \mid \mathbf{p}, \mathbf{p}_{\mathrm{VA}}^{(j)}\right)=\delta\left(\boldsymbol{\tau}-\frac{1}{c}\left\|\mathbf{p}-\mathbf{p}_{\mathrm{VA}}^{(j)}\right\|_{2}\right)$ and $\mathrm{p}(\boldsymbol{\alpha} \mid \boldsymbol{\tau})=\delta\left(\boldsymbol{\alpha}-\hat{\boldsymbol{\alpha}}_{\mathrm{LS}}\right)$, where $\hat{\boldsymbol{\alpha}}_{\mathrm{LS}}$ is the least-square solution describing the relation between complex amplitudes $\boldsymbol{\alpha}$ and the vector of delays $\tau$ at the agent position $\mathbf{p}$ (see (11)). $\delta(\cdot)$ denotes the Dirac-delta distribution. Then the estimation problem accounting for all anchors reduces to a MLE using (3) which is formulated as

$$
\hat{\mathbf{p}}_{\mathrm{ML}}=\underset{\mathbf{p}, \boldsymbol{\tau}, \boldsymbol{\alpha}}{\arg \max } \prod_{j=1}^{N_{\mathrm{A}}} \mathrm{p}\left(\mathbf{r}^{(j)} \mid \boldsymbol{\tau}, \boldsymbol{\alpha}, \mathbf{p}\right) .
$$

The actual MLE algorithm is described in section II-E. Independent of the used estimator, the likelihood has to be evaluated by applying the following steps:

1) To reduce the uncertainties in the geometry, a relocalization of the VA positions to $\hat{\mathbf{p}}_{k}^{(j)}$ of the $j$-th anchor is done beforehand by using a set of measurements with known agent positions. For detailed information about the re-localization process we refer the reader to [2].

2) With the pre-computed or re-localized set of VAs, the position dependent set of expected delays $\hat{\tau}_{k}^{(j)}(\mathbf{p})=$ $\frac{1}{c}\left\|\mathbf{p}-\hat{\mathbf{p}}_{k}^{(j)}\right\|_{2}$, is used to construct the signal matrix (4) by

$\hat{\mathbf{S}}=\left[\begin{array}{ccc}s\left(T_{s}-\hat{\tau}_{1}^{(j)}(\mathbf{p})\right) & \ldots & s\left(M T_{s}-\hat{\tau}_{1}^{(j)}(\mathbf{p})\right) \\ \vdots & & \vdots \\ s\left(T_{s}-\hat{\tau}_{K^{(j)}}^{(j)}(\mathbf{p})\right) & \ldots & s\left(M T_{s}-\hat{\tau}_{K^{(j)}}^{(j)}(\mathbf{p})\right)\end{array}\right]$

The expected delays $\tau_{k}^{(j)}(\mathbf{p})$ are refined by searching for the actual amplitude maximum in the received signal vector $\mathbf{r}^{(j)}$ in the window $\left[\hat{\tau}_{k}^{(j)}(\mathbf{p})-T_{p}, \hat{\tau}_{k}^{(j)}(\mathbf{p})+T_{p}\right]$.

3) Since the DM statistics are usually unknown, the covariance $\mathbf{C}$ has to be estimated from a sufficiently large set of measured signals $\left\{\mathbf{r}_{i}^{(j)}\right\}_{i=1}^{L}$ around the actual agent position. Using the signal matrix of (9) for the position hypothesis $\mathbf{p}_{i}$ of the $i$-th measurement, the template signal $\mathbf{r}_{\mathrm{det}, i}$ is constructed and further the co-variance matrix is estimated by

$$
\hat{\mathbf{C}}=\frac{1}{L} \sum_{i=1}^{L}\left(\mathbf{r}_{i}^{(\mathrm{al}, j)}-\mathbf{r}_{\mathrm{det}, i}\right)\left(\mathbf{r}_{i}^{(\mathrm{al}, j)}-\mathbf{r}_{\mathrm{det}, i}\right)^{\mathrm{H}} .
$$

Note, that the LOS propagation delay $\tau_{1, i}$ differences between the measurements has to be compensated to be able to average over the set of aligned measurements $\left\{\mathbf{r}_{i}^{(\mathrm{al}, j)}\right\}$ of the spatially close agent positions. In order to get a benchmark for the induced improvement of robustness and accuracy due to consideration of DM, we assume for the DM estimation known positions on a measurement grid around the actual agent position. In a realistic positioning application, this estimation has to be performed based on estimated positions and the corresponding signals hypotheses. However, this is considered as out of scope of this paper.

4) Using (9) and (10), the MLE of the complex amplitudes $\alpha$ reduces to a linear estimation problem. This means that the estimation is formulated as a weighted least square solution in the following form

$$
\hat{\boldsymbol{\alpha}}_{\mathrm{LS}}=\left(\hat{\mathbf{S}} \hat{\mathbf{C}}^{-1} \hat{\mathbf{S}}^{\mathrm{H}}\right)^{-1} \hat{\mathbf{S}} \hat{\mathbf{C}}^{-1} \mathbf{r}^{(j)} .
$$

The LHF is evaluated by inserting the estimated parameter $\hat{\mathbf{S}}$, $\hat{\mathbf{C}}$ and $\hat{\boldsymbol{\alpha}}_{\mathrm{LS}}$ into equation (3). 


\section{E. Implementation of the MLE}

Due to the fact that the LHF is highly multi-modal, nonGaussian and the measurement model is non-linear, a straightforward ML estimation is not applicable. Hence, we present a hybrid probabilistic-heuristic approach which combines a sequential importance re-sampling (SIR) particle filter (PF) with the concept of particle swarm global optimization (PSO) which is able to find the global maximum in the parameter space. In [8] probability model-based methods for global optimization are presented. A SIR particle filtering is suggested as a proper method for finding the global maximum. To explore the entire search space and find new candidate solutions, randomness has to be introduced in the maximization method. This can be realized: (i) in the re-sampling step of the PF by generating also new values for the particles instead of conventional resampling with replacement or (ii) via a state-space model which induces the exploration.

In our approach, we use a dynamic state-space model to explore the search-space. The state equation consists of two parts, the first one is described by a constant-velocity random walk model and the second part is responsible for the particle swarm behavior [7].

1) SIR Particle Filter: Particle filters represent a suboptimal sequential Monte Carlo method for solving non-linear and non-Gaussian sequential Bayesian state estimation problems which can not be computed in a closed form. In general, the Bayesian tracking problem is the recursive computation of a degree of belief of a hidden state, e.g. position and velocity $\mathbf{x}_{n}=\left[\mathbf{p}_{n}, \mathbf{v}_{n}\right]^{T}$, using measurement $\mathbf{r}_{n}$ at time-index $n$. Due to the fact that this paper deals with ML localization, not tracking, the index $n$ describes the evolution of the distribution as a function of time until convergence is reached, i.e. iterations use just one measurement, i.e. $\mathbf{r}_{1: n}=\mathbf{r}_{n}=\mathbf{r} \quad \forall n$. The state estimation is done in two consecutive stages using a firstorder hidden Markov model (HMM): (i) the prediction step which obtains the predicted posterior PDF $\mathrm{p}\left(\mathbf{x}_{n} \mid \mathbf{r}_{n}\right)$ using the Chapman-Kolmogorv equation [6]

$$
\mathrm{p}\left(\mathbf{x}_{n} \mid \mathbf{r}_{1: n-1}\right)=\int \mathrm{p}\left(\mathbf{x}_{n} \mid \mathbf{x}_{n-1}\right) \mathrm{p}\left(\mathbf{x}_{n-1} \mid \mathbf{r}_{1: n-1}\right) \mathrm{d} \mathbf{x}_{n-1},
$$

where $\mathrm{p}\left(\mathbf{x}_{n} \mid \mathbf{x}_{n-1}\right)$ is the state evolution probability, and (ii) the update step which is solved via Bayes' rule

$$
\mathrm{p}\left(\mathbf{x}_{n} \mid \mathbf{r}_{1: n}\right)=\frac{\mathrm{p}\left(\mathbf{r}_{n} \mid \mathbf{x}_{n}\right) \mathrm{p}\left(\mathbf{x}_{n} \mid \mathbf{r}_{1: n-1}\right)}{\int \mathrm{p}\left(\mathbf{r}_{n} \mid \mathbf{x}_{n}\right) \mathrm{p}\left(\mathbf{x}_{n} \mid \mathbf{r}_{1: n-1}\right) \mathrm{d} \mathbf{x}_{n}},
$$

where $\mathrm{p}\left(\mathbf{r}_{n} \mid \mathbf{x}_{n}\right)=\mathrm{p}\left(\mathbf{r}_{n} \mid \boldsymbol{\tau}, \boldsymbol{\alpha}, \mathbf{p}_{n}\right)$ is the measurement LHF. For solving (12) and (13), particle filters use a finite set of weighted samples, i.e. particles $\left\{\mathbf{x}_{n}^{i}, a_{n}^{i}\right\}_{i=1}^{N}$, to approximate the involved PDFs. The particles are sampled from an importance distribution $\mathbf{x}_{n}^{i} \sim \mathrm{q}\left(\mathbf{x}_{n} \mid \mathbf{x}_{n-1}, \mathbf{r}_{n}\right)$ and their weights $a_{n}^{i}$ are computed in each iteration with

$$
a_{n}^{i}=a_{n-1}^{i} \frac{\mathrm{p}\left(\mathbf{r}_{n} \mid \mathbf{x}_{n}^{i}\right) \mathrm{p}\left(\mathbf{x}_{n}^{i} \mid \mathbf{x}_{n-1}^{i}\right)}{\mathrm{q}\left(\mathbf{x}_{n}^{i} \mid \mathbf{x}_{n-1}^{i}, \mathbf{r}_{n}\right)}, \quad \sum_{i=1}^{N} a_{n}^{i}=1 .
$$

A simple choice of importance distribution is to take the state evolution probability $\mathrm{q}\left(\mathbf{x}_{n} \mid \mathbf{x}_{n-1}, \mathbf{r}_{n}\right)=\mathrm{p}\left(\mathbf{x}_{n} \mid \mathbf{x}_{n-1}\right)$. To reduce the degeneration of the particles, a re-sampling step is introduced after every iteration. In this step, particles are drawn according to their weight which means that particle states $\mathbf{x}_{n}^{i}$ with high weight $a_{n}^{i}$ are duplicated more often than particles with lower weights. The particle weights after every re-sampling step are set to $a_{n}^{i}=1 / N$. So, the computation of the weights simplifies to $a_{n}^{i} \propto \mathrm{p}\left(\mathbf{r}_{n} \mid \mathbf{x}_{n}^{i}\right)$.

2) PF-PSO: In PSO the particles are generated randomly and by iterative updates of the positions using a cost function. The particles learn from their own cost-measure and the costmeasure of the other particles introducing swarm behavior. In our case, the particles in the swarm gain a velocity in direction of the global maximum, so that the PSO is able to jump out of local maxima and find the global optimum. This swarm behavior is integrated in the PF by the state transition equation [7] which is described by

$$
\begin{aligned}
\mathbf{x}_{n} & =\mathbf{F} \mathbf{x}_{n-1}+u_{1}\left[\begin{array}{c}
\mathbf{p}_{\mathrm{b}}^{i \text { best }}-\mathbf{p}_{n-1} \\
w_{v}\left(\mathbf{p}_{\mathrm{b}}^{\text {ibest }}-\mathbf{p}_{n-1}\right)
\end{array}\right] \\
& +u_{2}\left[\begin{array}{c}
\mathbf{p}_{\mathrm{b}}^{\mathrm{g}}-\mathbf{p}_{n-1} \\
w_{v}\left(\mathbf{p}_{\mathrm{b}}^{\mathrm{g}}-\mathbf{p}_{n-1}\right)
\end{array}\right]+\mathbf{G n}_{\mathrm{acc}} .
\end{aligned}
$$

Here,

$$
\mathbf{F}=\left[\begin{array}{cccc}
1 & 0 & w_{v} & 0 \\
0 & 1 & 0 & w_{v} \\
0 & 0 & w_{v} & 0 \\
0 & 0 & 0 & w_{v}
\end{array}\right] \quad \text { and } \quad \mathbf{G}=\left[\begin{array}{cc}
\frac{w_{v}^{2}}{2} & 0 \\
0 & \frac{w_{v}^{2}}{2} \\
w_{v} & 0 \\
0 & w_{v}
\end{array}\right]
$$

are the state transition matrix and the noise weighting matrix, $w_{v}$ is the velocity weight, $u_{1}$ and $u_{2}$ are random control factors drawn from a uniform distribution $\mathcal{U}(0,1), \mathbf{n}_{\text {acc }}$ is a zeromean Gaussian driving acceleration noise with co-variance matrix $\sigma_{\mathrm{acc}}^{2} \mathbf{I}_{2} \cdot \mathbf{p}_{\mathrm{b}}^{\text {ibest }}$ and $\mathbf{p}_{\mathrm{b}}^{\mathrm{g}}$ denote the maximum of the set of particles in the current iteration $n$ and the global maximum of the past iterations, respectively. The algorithm starts with uniformly distributed particles over the entire search space. For these candidates the likelihood function is evaluated and the global maximum of the LHF $\mathrm{L}_{\mathrm{g}}$ and the corresponding position $\mathbf{p}_{\mathrm{b}}^{\mathrm{g}}=\arg \max _{\mathbf{p}_{n}^{i}} \mathrm{p}\left(\mathbf{r} \mid \boldsymbol{\tau}, \boldsymbol{\alpha}, \mathbf{p}_{n}^{i}\right)$ are computed. Further, the current maximum of the particles $\mathrm{L}^{\text {ibest }}=\mathrm{L}_{\mathrm{g}}$ and also the corresponding position $\mathbf{p}_{\mathrm{b}}^{i \text { best }}=\mathbf{p}_{\mathrm{b}}^{\mathrm{g}}$ are set to the global values. Then, the state $\mathbf{x}_{n}^{i}$ (prediction step) and the according weights $a_{n}^{i}$ (measurement update) of the particles are evaluated iteratively for a defined number of iterations. After each measurement update and before the re-sampling step is executed, the maximum of the current particles $\mathrm{L}^{i \text { best }}$ at position $\mathbf{p}_{\mathrm{b}}^{\text {ibest }}$ is computed and accepted as new global maximum if $\mathrm{L}^{i \text { best }}>\mathrm{L}_{\mathrm{g}}$.

\section{Performance Evaluation}

\section{A. Setup}

1) Measurement: The measurement scenario is illustrated in Fig. 1. We have used an M-sequence UWB channel sounder from Ilmsens (See: www.ilmsens.com) to record the UWB channel impulse responses (CIRs). The device is equipped with one TX and two RXs channels. We have used self made 
dipole antennas having an almost isotropic radiation pattern in the horizontal plane. In the measurement scenario, the anchors correspond to the RX and the agent to the TX. The anchors are placed at positions $\mathbf{p}_{1}^{(1)}=[0.5,7]^{\mathrm{T}}$ and $\mathbf{p}_{1}^{(2)}=[5.2,3.2]^{\mathrm{T}}$, respectively. The measurement trajectory of the agent consists of 220 points spaced by $5 \mathrm{~cm}$. In addition to this, for every agent position on the trajectory, 25 measurements have been recorded with the agent placed according to the grid shown in Fig. 1. These additional measurements serve for the estimation of DM in the vicinity of the agent trajectory point. To obtain the UWB-CIR, the received signal and the transmitted M-sequence are cross-correlated. The bandwidth of the channel sounder corresponds to the FCC UWB range from $3.1-10.6 \mathrm{GHz}$. A raised cosine filter $s(t)$ with roll-off factor $\beta_{\mathrm{r}}=0.5$ and an effective pulse duration of $T_{P}=1 / B$ is used to select a specific sub-band with bandwidth $B=2 \mathrm{GHz}$ at center-frequency $f_{c}=7 \mathrm{GHz}$. To get the complex-valued baseband signal, a down conversion is performed.

2) Estimation: To evaluate the LHF in (2), we used two approaches: (i) Grid based MAP: The LHF is evaluated over a $40 \times 40 \mathrm{~cm}$ rectangle around the true agent position with resolution of $1 \times 1 \mathrm{~cm}^{2}$ and the highest mode corresponds to the MLE given in (8); (ii) PF-PSO: A sufficiently large number of particles, i.e. $N=2000$, is uniformly distributed over the whole room. The LHF is evaluated for $N_{\text {iter }}=8$ iterations following the description given in Sec. II-E1, where the initial acceleration process noise $\sigma_{\text {acc }}=8 \frac{\mathrm{cm}}{\mathrm{s}^{2}}$ and the initial velocity weight $w_{v}=0.0125$. Since the LHF has very narrow modes, the weights $a_{n}^{i}$ are compressed with a roughening factor which means that the exponent of the LHF was divided by this factor. The initial value of the roughening factor was set to value of 100. During the iterations of the PF-PSO filter, these three parameters were linear decreased to simulate an annealing process, whereas the roughening factor was decreased until it reaches 1 . To enhance robustness of the point-estimate $\hat{\mathbf{p}}$ of the MLE, we computed the median over the entire set of particles at each trajectory point. To generate the error CDFs we performed Monte-Carlo simulations averaged over $30^{1}$ dynamic model noise realizations.

\section{B. Discussion of Performance Results}

1) UWB-CIR measurements and DM estimate: In Fig. 2 the CIR of the signal received at anchor 2 and agent position $\mathbf{p}=$ $[2.2,6.6]^{\mathrm{T}}$ is shown. The direct LOS path is clearly visible together with MPCs corresponding to specular reflections of first and second order. Their expected delays are illustrated in the figure as dashed gray vertical lines. One can observe that they fit the model quite well. The amplitude and phase of the emitted wave change according to the reflection coefficient of the wall. This is not modeled by the VA and we observe that not all expected VAs have a significant contribution.

2) Measurements, Position Likelihood, and CRLB: In Fig. 3 , the likelihood (3) is shown in log-domain evaluated for the

\footnotetext{
${ }^{1}$ We observed that this number of used Monte-Carlo simulations leads to a steady state outcome suggesting that it is sufficiently high.
}

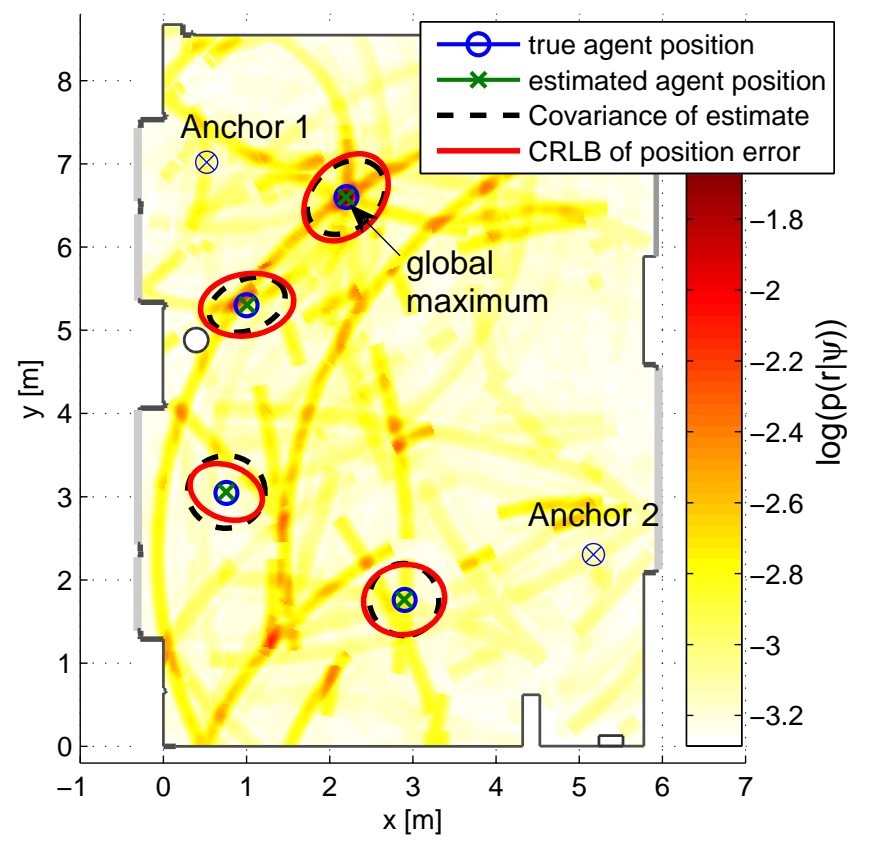

Fig. 3. Likelihood evaluated over the whole room. The anchors are placed at $\mathbf{p}_{1}^{(1)}=[0.5,7]^{\mathrm{T}}$ and $\mathbf{p}_{1}^{(2)}=[5.2,3.2]^{\mathrm{T}}$, the agent is placed at $\mathbf{p}=[2.2,6.6]^{\mathrm{T}}$. The global maximum at position $\hat{\mathbf{p}}=[2.189,6.599]^{\mathrm{T}}$ matches well with the true agent position. Additionally, the estimated agent position, the co-variance and the CRLB are shown with hundred-fold standard deviation for a few other agent positions.

whole room for a measured CIR $\mathbf{r}^{(j)}$ between the agent located at position $\mathbf{p}=[2.2,6.6]^{\mathrm{T}}$ and the two anchors. In the figure, the multi-modality of the LHF is clearly visible. The global maximum at $\hat{\mathbf{p}}=[2.189,6.599]^{\mathrm{T}}$ matches the true position of the agent very well. The radii of the arcs with a high likelihood correspond to the delays of the LOS and VAs visible in the measurements. In the figure, we also plot the estimated positions and co-variances (estimated from particles) for a few trajectory points together with the computed CRLB of the position error. Both error ellipses are plotted with hundredfold standard deviation. The orientation of the error ellipses depends on the geometry of the room and the positions of the anchors and the agent involved. We observe that the orientation and size of the CRLB error ellipses fit well with the estimated co-variance ellipses. Small deviations can be explained by the fact that the co-variance has to be estimated from a set of measurements rather than from a single measurement. The same holds for the estimation of parameters needed for the computation of the CRLB, c.f. (5). So, this comparison gives just an approximate comparison between the CRLB and the co-variance of the position estimate.

3) Positioning performance: In Fig. 4, the cumulative distribution functions (CDFs) of the position error are shown for the grid-based MAP and the MLE (PF-PSO method). These CDFs include data from all 220 agent positions using only anchors 1 and 2 individually and in combination (top to bottom). The CDF plots for grid-based MAP and MLE 


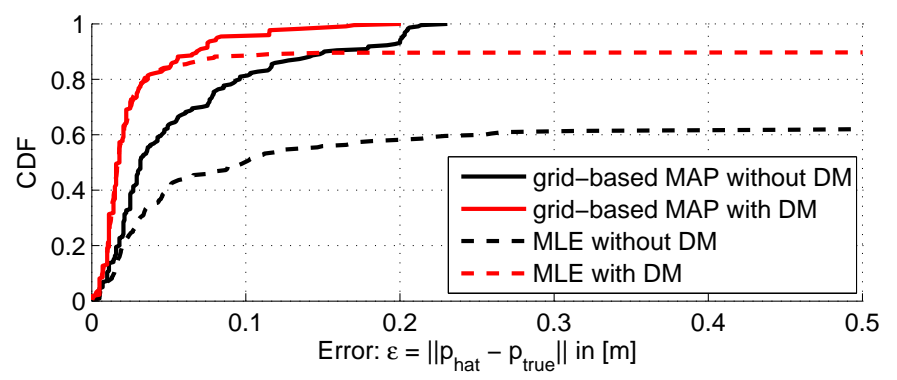

(a) Anchor 1

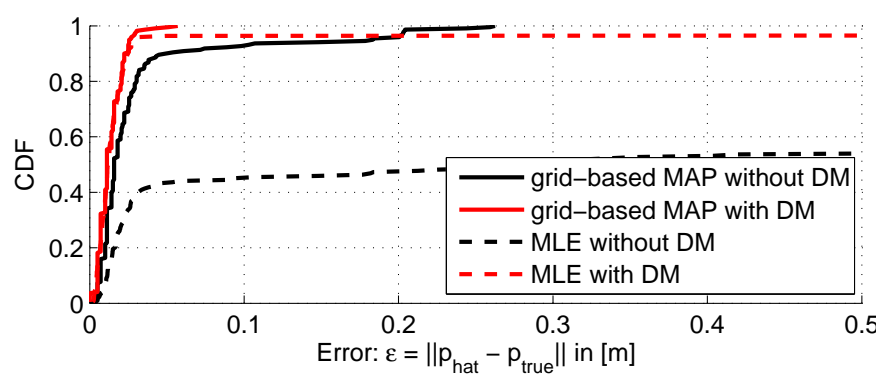

(b) Anchor 2

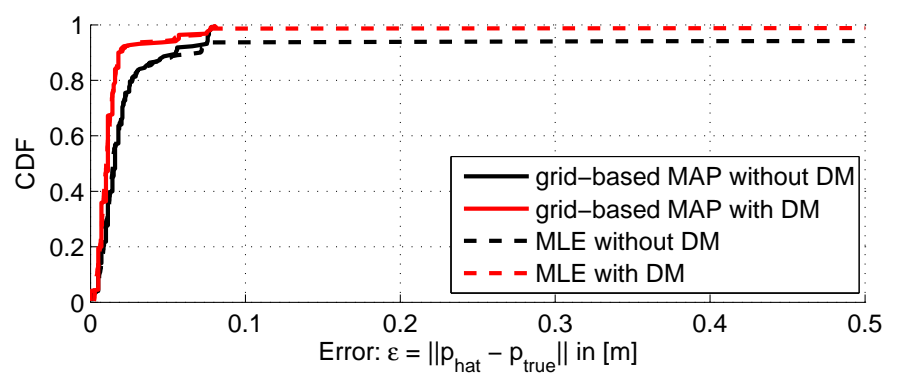

(c) Anchor 1 and 2

Fig. 4. CDFs for the grid-based MAP and MLE methods, with and without considering DM in the LHF considering only anchor 1 (a), anchor 2 (b) or the combination of both (c).

"without DM" neglect the knowledge of DM, i.e. $\mathbf{C}^{(j)}=$ $N_{0}^{(j)} \mathbf{I}_{M}$ in the likelihood (3). Then the signal model reduces to the deterministic MPC plus additional AWGN (c.f. (1)). We observe that for both methods, the knowledge of DM results in a reduction of the position errors. Also, the occurrence of outliers is reduced when considering DM, which demonstrates the benefit of the suggested signal model and can be seen as a measure of higher robustness. If only anchor 2 is active, the position error is below $2.5 \mathrm{~cm}$ in $90 \%$ of the estimates for the grid based MAP method considering DM and $5.5 \mathrm{~cm}$ without DM knowledge.

The MLE with DM knowledge is also below $2.5 \mathrm{~cm}$ in $90 \%$ of the estimates while it reaches a similar accuracy in only $40 \%$ of the cases without DM. For all combinations of the involved anchors without DM knowledge, the performance of the MLE is much worse. The reasons for this are twofold: First, the model is less accurate, thus modes of the LHF at the wrong position are too optimistic which leads more often to a convergence of the MLE in the wrong mode. The second issue is of numerical nature. Due to the more probable model- mismatch of the signal model, the values of the log-LHF are even (much) smaller and also exhibit a larger dynamic range. Hence, the LHF is more skewed which leads to the requirement of more particles for the MLE. If anchor 1 is active, the positioning error shows the same tendency, but the values are increased. This suggests that the location of anchor 1 is less suited for positioning along the agent trajectory compared to the location of anchor 2 .

Since information available through measurements never increases the uncertainty, the error is the smallest when both anchors are active. This is independent of the signal model used (with or without DM). The lowest error is achieved if DM is taken into account. Then the resulting error remains below $1.9 \mathrm{~cm}$ for both methods in $90 \%$ of the position estimates. Note that the bandwidth used is $2 \mathrm{GHz}$, yielding a delay resolution of $15 \mathrm{~cm}$.

\section{CONCLUSION AND OUTLOOK}

In this paper we have shown that the proposed MLE algorithm for multipath-assisted indoor positioning is working with real channel measurement in a robust and accurate sense. Due to the rich geometric information contained in the MPCs, positioning is possible with only one anchor. Depending on the environment this is also true for non-LOS scenarios, in principle. In-depth verification of this is part of future work. However, in a LOS scenario considering DM we achieved a position error of less than $2.5 \mathrm{~cm}$ in $90 \%$ of the estimates for only one active anchor. The results show that the knowledge of DM leads to significant improvement of robustness and accuracy of the estimation scheme. However, the demonstrated DM estimation from a set of measurements with optimal position knowledge has to be seen as an ideal benchmark for the benefit of using DM. In ongoing work we learn the DM statistics during tracking of an agent moving through the room to establish a more practical estimator.

\section{REFERENCES}

[1] A. Molisch, "Ultra-wide-band propagation channels," Proceedings of the IEEE, vol. 97, no. 2, pp. 353-371, Feb. 2009.

[2] P. Meissner and K. Witrisal, "Analysis of position-related information in measured UWB indoor channels," in Antennas and Propagation (EUCAP), 2012 6th European Conference on, March 2012, pp. 6-10.

[3] - "Multipath-assisted single-anchor indoor localization in an office environment," in Systems, Signals and Image Processing (IWSSIP), 2012 19th International Conference on, April 2012, pp. 22-25.

[4] Y. Shen and M. Win, "Fundamental limits of wideband localization; part I: A general framework," Information Theory, IEEE Transactions on, vol. 56, no. 10, pp. 4956-4980, Oct. 2010.

[5] K. Witrisal and P. Meissner, "Performance bounds for multipath-assisted indoor navigation and tracking (MINT)," in Communications (ICC), 2012 IEEE International Conference on, June 2012, pp. 4321-4325.

[6] M. Arulampalam, S. Maskell, N. Gordon, and T. Clapp, "A tutorial on particle filters for online nonlinear/non-gaussian bayesian tracking," Signal Processing, IEEE Transactions on, vol. 50, no. 2, pp. 174-188, Feb 2002.

[7] G. Tong, Z. Fang, and X. Xu, "A particle swarm optimized particle filter for nonlinear system state estimation," in Evolutionary Computation, 2006. CEC 2006. IEEE Congress on, 2006, pp. 438-442.

[8] J. Hu, Y. Wang, E. Zhou, M. Fu, and S. Marcus, "A survey of some modelbased methods for global optimization," in Optimization, Control, and Applications of Stochastic Systems, ser. Systems \& Control: Foundations \& Applications. Birkhäuser Boston, 2012, pp. 157-179. 